\title{
The effects of nonreinforced and randomly reinforced stimulus preexposure on conditioned suppression in rats
}

\author{
MARY SHORE LOGAN and PAUL SCHNUR \\ Western College, Oxford, Ohio 45056
}

\begin{abstract}
An experiment is reported in which two groups of rats received stimulus familiarization training prior to classical conditioning. Group NRPE (nonreinforced preexposure) received 24 preexposures to the conditioned stimulus (CS) on each of 4 days; Group RRPE (randomly reinforced preexposure) received the same number of CS preexposures but interspersed with uncorrelated presentations of a 2-mA footshock; Group NPE (no preexposure) was a control group which received no preexposures. All groups then received on-the-baseline classical conditioning trials, and suppression of the operant baseline in the presence of the CS was monitored. Group NRPE was significantly retarded in acquisition of conditioned suppression compared to Groups RRPE ( $p<.029)$ and NPE $(p<.014)$, but the latter two groups did not differ from each other. These results are in opposition to earlier findings (Mackintosh, 1973) and are discussed in terms of stimulus salience.
\end{abstract}

Many theories of associative learning propose that the associability of a stimulus with a reinforcer depends upon the relative salience or attention value of the conditioned stimulus (e.g., Lovejoy, 1968; Rescorla \& Wagner, 1972; Sutherland \& Mackintosh, 1971; Zeaman \& House, 1963). Other things being equal, high salience facilitates associative learning, while low salience retards it. In a recent theory of selective attention, Mackintosh (1975) proposed that the salience of a stimulus increases when that stimulus predicts a change in the probability of reinforcement already predicted by other stimuli, and, conversely, that the salience of a stimulus decreases when that stimulus signals no change in the probability of reinforcement predicted by other stimuli. A similar proposal has been made by Lubow and his colleagues (Lubow, Alek, \& Arzy, 1975; Lubow, Rifkin, \& Alek, 1976; Lubow, Schnur, \& Rifkin, 1976).

Among the findings relevant to this hypothesis are those concerning the debilitative effects of stimulus familiarization on conditioning. A substantial body of evidence shows that nonreinforced stimulus preexposure (NRPE) as well as randomly reinforced stimulus preexposure (RRPE) retard subsequent conditioning (Lubow, 1973; Mackintosh, 1973). Both procedures familiarize subjects with the conditioned stimulus (CS), but only the latter simultaneously exposes subjects to uncorrelated presentations of the unconditioned stimulus (UCS) or reinforcer. In both familiarization procedures, the conditioned stimulus predicts no change in the prob-

The research reported in this article was supported by a grant to the second author from the National Science Foundation, GB-33682. The assistance of Dona Garrett Gardner, Jay Harburger, and Jill Rawnsley is gratefully acknowledged. The paper is sponsored by R. E. Lubow. Mary Shore Logan is now at the University of Pennsylvania and Paul Schnur is now at Fordham University/Lincoln Center. ability of reinforcement already predicted by other stimuli (e.g., background cues). Therefore, one might expect to observe equivalent effects on conditioning following NRPE and RRPE (Mellgren \& Ost, 1971). On the other hand, since during RRPE the stimulus predicts no change in a specific reinforcer, while in NRPE the stimulus simply predicts no change, one might expect to observe differential effects on conditioning following NRPE and RRPE. According to Mackintosh (1973, 1975), the general pattern is for RRPE to produce more serious and longer lasting retardation of conditioning than NRPE.

One interpretation of this difference, favored by Mackintosh (1975), is that the decrease in salience engendered by RRPE is specific to the reinforcer, while that engendered by NRPE is not reinforcer specific. In fact, Mackintosh (1973) has shown that, under some conditions, the effects of RRPE are reinforcer specific, durable, and relatively strong, and those of NRPE are reinforcer nonspecific, transient, and relatively weak. The present experiment compared the effects of RRPE and NRPE on conditioned suppression in rats.

\section{METHOD}

\section{Subjects}

The subjects were 12 male albino rats of the Sprague-Dawley strain (Holtzman Company), approximately 70-80 days of age at the start of the experiment. They were housed singly in wire mesh cages. For the first week after arrival at the lab, feeding was ad lib; during the second week and for the remainder of the experiment, the subjects were fed $12 \mathrm{~g}$ of food daily on a 24-h schedule, reducing them to approximately $80 \%$ of their normal body weight. The rats were weighed and fed 5-10 min after each experimental session. Water was freely available at all times.

\section{Apparatus}

The apparatus consisted of two rat test chambers (BRSForinger) enclosed in light and sound attenuating cubicles (BRS- 


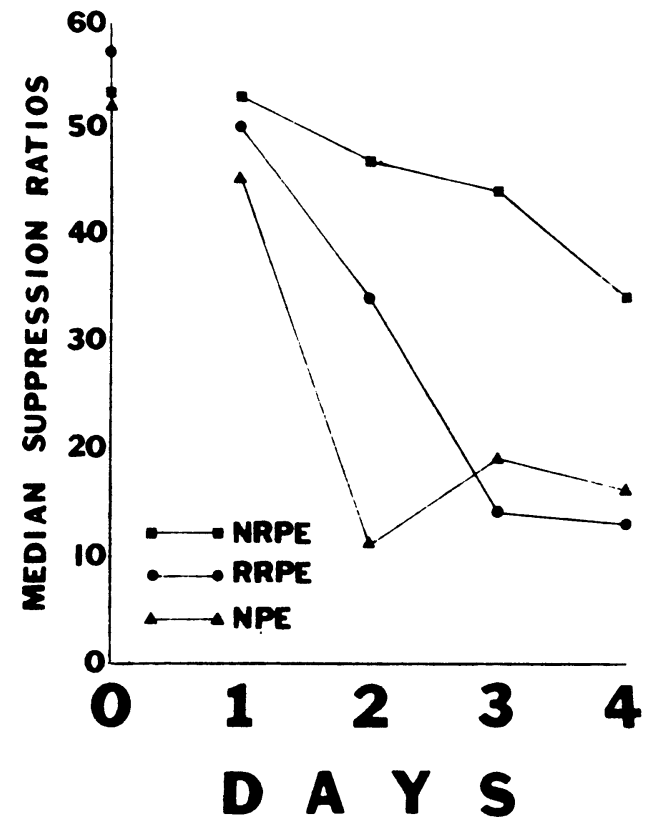

Figure 1. Median suppression ratios for all groups as a function of days of acquisition. The points plotted above zero on the abscissa are median suppression ratios for the first trial of acquisition.

Foringer). A response lever and food hopper were located on one end panel of each chamber. Barpress responses were reinforced with $45-\mathrm{mg}$ Noyes pellets. The chamber floors consisted

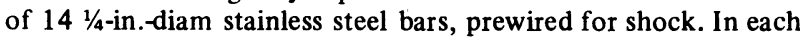
cubicle, a ventilator fan provided a 75-dB SPL masking noise. A BRS-Foringer audio oscillator produced the tone CS $(1,000 \mathrm{~Hz}$ at $71 \mathrm{~dB}$ SPL). A 2-mA electric shock UCS applied to the grid floors was produced by a BRS-Foringer generator/scrambler (Model SGS-009). Recording and programming equipment were located in an adjacent room.

\section{Procedure}

All animals were given one 2-h experimental session daily. The first six sessions were devoted to barpress training. On Day 1, subjects were magazine trained and shaped: One food pellet was delivered on a variable interval (VI) 1 -min schedule and each barpress yielded an additional pellet. On Day 2 , subjects responded for the first hour on a VI 1-min schedule of reinforcement and for the second hour on a VI 2-min schedule. On Days 3-6, the reinforcement schedule was VI 2 min.

On Days 7-10, the bars were removed from the boxes and off-the-baseline preexposure procedures were delivered. For this phase of the experiment, subjects were randomly assigned to one of three groups. Group NRPE (nonreinforced preexposure) received 2-min tone CSs presented on a fixed interval (FI) 5 -min schedule. A $2-\mathrm{h}$ session thus contained $24 \mathrm{CS}$ preexposures. For Group RRPE (randomly reinforced preexposure), CS presentations were the same as for Group NRPE, but, in addition, 2-mA shocks of $1-\mathrm{sec}$ duration were delivered randomly and independently of the CS throughout the session. Shock was equiprobable in the presence and absence of the CS: For each 2 min of the session, a shock occured with $p=.40$. The third group of animals, Group NPE (no preexposure) was given neither CS nor shock preexposure, but was placed in the apparatus for the length of the experimental session.

Following preexposure, the bars were replaced and subjects were allowed 1 day for recovery of the barpress response. Reinforcement was delivered on a VI 2-min schedule.
Days 12-15 consisted of on-the-baseline classical conditioning. Four CS-UCS trials per day were superimposed on the VI baseline. Trials were presented at $20,50,70$, and $100 \mathrm{~min}$ into the experimental session. The 2-min tone CS terminated in the onset of the 1-sec 2-mA footshock. Acquisition procedures were identical for all groups.

\section{Response Measure}

The dependent variable was suppression of the barpress response in the presence of the CS. A suppression ratio of the form, $A / A+B$, was calculated, where $A$ is the number of responses during the $C S$ and $B$ is the number of responses in the 2-min period immediately preceding CS onset.

\section{RESULTS}

A Kruskal-Wallis one-way analysis of variance indicated that there were no differences among groups in response rate during the $2-\min$ pre-CS periods $(\mathrm{H}=1.96$, $\mathrm{p}>.10)$.

Figure 1 shows median suppression ratios for all groups as a function of days of acquisition. It is evident that both the nonpreexposed control group (NPE) and the randomly reinforced preexposure group (RRPE) conditioned more readily than did the nonreinforced preexposure group (NRPE). A Kruskal-Wallis one-way analysis of variance indicated that the groups differed reliably during acquisition $(\mathrm{H}=6.73, .011<\mathrm{p}<.049$ ). In addition, Mann-Whitney U-tests revealed that Group NRPE conditioned more slowly than either Group NPE $\quad(U=0, \quad p<.014, \quad$ one-tailed $)$ or Group RRPE $(U=1, p<.029$, one-tailed $)$. Figure 1 suggests that, compared to nonpreexposure, randomly reinforced CS preexposure initially retarded learning. However, the effect was only transient, and, overall, Groups NPE and RRPE did not differ significantly $(U=6, p>.05)$.

\section{DISCUSSION}

The results of the present experiment confirm much previous work in showing that nonreinforced CS preexposure retards subsequent conditioning (Lubow, 1973). Moreover, the present experiment demonstrates that the effects of nonreinforced preexposure are not equivalent to those of randomly reinforced preexposure (cf. Mellgren \& Ost, 1971). Indeed, NRPE has effects which are stronger and more durable than those of RRPE. If the present results are to be interpreted in terms of decreases in stimulus salience, one may reasonably conclude that stimuli which predict no change lose salience more than stimuli which predict no change in the occurrence of a specific reinforcer. Perhaps uncorrelated UCS presentations offset some of the decrease in salience produced by nonreinforced preexposure. Such an effect is, in fact, anticipated by conditioned attention theory (Lubow et al., 1975) and by a recent information processing theory of habituation (Wagner, in press).

Whatever the ultimate explanation of changes in stimulus salience may be, a prior question concerns the fact that Mackintosh (1973) has found RRPE to produce a greater decrement in conditioning than NRPE, an effect opposite to that reported here. Such a marked discrepancy between the outcomes of studies comparing ostensibly similar familiarization procedures implicates the contributions of other variables to 
changes in stimulus salience. One such variable is the density of stimulus events presented during NRPE and RRPE (Kremer, 1971). Kremer (1971) found that the effect of RRPE on subsequent conditioning depended upon how many CSs and UCSs were presented in a 2 -h session. When there were 202 -min CSs and $20 \mathrm{1} / 2$-sec UCSs per familiarization session (high density), subsequent conditioning was facilitated compared to either NPE or NRPE (20 CSs per session). When there were only four 2-min CSs and four $1 / 2$-sec UCSs per session (low density), subsequent conditioning was retarded compared to either NPE or NRPE (four CSs per session). The latter result is the one obtained by Mackintosh (1973), who presented eight 20-sec CSs in a 30-min session. Thus, when the CS occupies a small proportion of the total familiarization session (Kremer, 1971, Experiment 2; Mackintosh, 1973), RRPE retards subsequent conditioning. However, when the CS occupies a large proportion of the total familiarization session (Kremer, 1971, Experiment 1), RRPE facilitates subsequent conditioning as compared to NRPE and NPE. In the present study, the density of stimulus events was high and, again, RRPE facilitated conditioning relative to NRPE, though not as compared to NPE.

Although the density of stimulus events seems to offer a partial explanation of the relative effects of RRPE, NRPE, and NPE, numerous questions remain unresolved. For example, the high-density schedule used by Kremer (1971, Experiment 1), facilitated conditioning following RRPE as compared to NRPE and NPE; whereas, in the present study, Group RRPE conditioned faster than Group NRPE, but not faster than Group NPE. In fact, Group RRPE was initially retarded as compared to Group NPE. In addition, there are two stimulus densities-that of the CS and that of the UCS-whose effects must be independently considered. In the studies reviewed above, these two were completely confounded. As Kremer (1971) has noted, high- and low-density schedules differ in terms of: the proportion of the familiarization session filled by CS events, the proportion of the session filled by UCS events, the number of fortuitous contiguous pairings of CS and UCS, the mean CS-UCS interval, etc. The extent to which each of these variables contributes to changes in stimulus salience remains to be determined. Finally, other parametric differences between experiments may have contributed to the discrepant results. Mackintosh (1973) used pigeons in an autoshaping paradigm; Kremer (1971) used hooded rats in a conditioned suppression task similar to the one reported herein. In addition, various stimulus and training parameters differed between the latter two studies.

Thus, the present experiment demonstrates that a stimulus familiarized in the absence of reinforcement has different functional properties than a stimulus familiarized in the presence of uncorrelated reinforcement. Furthermore, under the conditions of the present experiment, the former procedure interferes with subsequent conditioning more than the latter. It seems clear that an understanding of changes in stimulus salience requires a more extensive analysis of the procedures in which both CS and UCS are preexposed concurrently.

\section{REFERENCES}

Kremer, E. F. Truly random and traditional control procedures in CER conditioning in the rat. Journal of Comparative and Physiological Psychology, 1971, 76, 441-448.

Lovejoy, E. Attention in discrimination learning. San Francisco: Holden-Day, 1968.

Lubow, R. E. Latent inhibition. Psychological Bulletin, 1973, 79, 398-407.

Lubow, R. E., Alek, M., \& Arzy, J. Behavioral decrement following stimulus preexposure: Effects of number of preexposures, presence of a second stimulus, and interstimulus interval in children and adults. Journal of Experimental Psychology: Animal Behavior Processes, 1975, 1, 178-188.

Lubow, R. E., Rifkin, B., \& Alek, M. The context effect: The relationship between stimulus preexposure and environmental preexposure determines subsequent learning. Journal of Experimental Psychology: Animal Behavior Processes, 1976, 2, 38-47.

Lubow, R. E., Schnur, P., \& Rifkin, B. Latent inhibition and conditioned attention theory. Journal of Experimental Psychology: Animal Behavior Processes, 1976, 2, 163-174.

MaCKintosh, N. J. A theory of attention: Variations in the associability of stimuli with reinforcement. Psychological Review, 1975, 82, 276-298.

Mackintosh, N. J. Stimulus selections: Learning to ignore stimuli that predict no change in reinforcement. In R. A. Hinde \& J. Stevenson-Hinde (Eds.), Constraints on learning. London: Academic Press, 1973.

Mellgren, R. L., \& Ost, J. W. P. Discriminative stimulus preexposure and learning of an operant discrimination in the rat. Journal of Comparative and Physiological Psychology, 1971, 77, 179-187.

Rescorla, R. A., \& WAgner, A. R. A theory of Pavlovian conditioning: Variations in the effectiveness of reinforcement and nonreinforcement. In A. H. Black \& W. F. Prokasy (Eds.), Classical conditioning II: Current research and theory. New York: Appleton-Century-Crofts, 1972.

Sutherland, N. S., \& Mackintosh, N. J. Mechanisms of animal discrimination learning. New York: Academic Press, 1971.

WAGNer, A. R. Priming in STM: An information processing mechanism for self-generated or retrieval-generated depression in performance. In T. J. Tighe \& R. N. Leaton (Eds.), Habituation: Perspectives from child development, animal behavior, and neurophysiology. Hillsdale, N.J: Lawrence Erlbaum, in press.

Zeaman, D., \& House, B. J. The role of attention in retardate discrimination learning. In N. R. Ellis (Ed.), Handbook of mental deficiency: Psychological theory and research. New York: McGraw-Hill, 1963.

(Received for publication June 21, 1976.) 